\title{
Acellular human lung scaffolds to model lung disease and tissue regeneration
}

\author{
Sarah E. Gilpin ${ }^{1,2}$ and Darcy E. Wagner (103,4,5 \\ Affiliations: ${ }^{1}$ Laboratory for Organ Engineering and Regeneration, Center for Regenerative Medicine, \\ Massachusetts General Hospital, Boston, MA, USA. ${ }^{2}$ Harvard Medical School, Boston, MA, USA. ${ }^{3}$ Lund \\ University, Dept of Experimental Medical Sciences, Lung Bioengineering and Regeneration, Lund, Sweden. \\ ${ }^{4}$ Wallenberg Center for Molecular Medicine, Lund University, Lund, Sweden. ${ }^{5}$ Stem Cell Centre, Lund \\ University, Lund, Sweden.
}

Correspondence: Darcy E. Wagner, Dept of Experimental Medical Sciences, Wallenberg Center for Molecular Medicine and Lund Stem Cell Centre, Lund University, BMC C12, S-221 84 Lund, Sweden.

E-mail: darcy.wagnerlamed.lu.se

@ERSpublications

Acellular human lung scaffolds can be used as diverse tools to study lung disease and tissue regeneration ex vivo http://ow.ly/ZS0l30k9MEH

Cite this article as: Gilpin SE, Wagner DE. Acellular human lung scaffolds to model lung disease and tissue regeneration. Eur Respir Rev 2018; 27: 180021 [https://doi.org/10.1183/16000617.0021-2018].

ABSTRACT Recent advances in whole lung bioengineering have opened new doors for studying lung repair and regeneration ex vivo using acellular human derived lung tissue scaffolds. Methods to decellularise whole human lungs, lobes or resected segments from normal and diseased human lungs have been developed using both perfusion and immersion based techniques. Immersion based techniques allow laboratories without access to intact lobes the ability to generate acellular human lung scaffolds. Acellular human lung scaffolds can be further processed into small segments, thin slices or extracellular matrix extracts, to study cell behaviour such as viability, proliferation, migration and differentiation. Recent studies have offered important proof of concept of generating sufficient primary endothelial and lung epithelial cells to recellularise whole lobes that can be maintained for several days ex vivo in a bioreactor to study regeneration. In parallel, acellular human lung scaffolds have been increasingly used for studying cell-extracellular environment interactions. These studies have helped provide new insights into the role of the matrix and the extracellular environment in chronic human lung diseases such as chronic obstructive pulmonary disease and idiopathic pulmonary fibrosis. Acellular human lung scaffolds are a versatile new tool for studying human lung repair and regeneration ex vivo.

\section{Introduction}

Chronic lung diseases such as chronic obstructive pulmonary disease are currently the third leading cause of death worldwide. Other chronic respiratory diseases, such as interstitial lung disease (ILD) or pulmonary arterial hypertension, and genetic conditions, such as $\alpha_{1}$-antitrypsin disorder and cystic fibrosis, are devastating lung diseases with no cure. At end-stage disease, transplantation is the only option. In addition to a shortage of suitable donor lungs, lung transplantation is further complicated by high rates of both acute and chronic rejection. As a result of these challenges, the current 5 -year survival rate is $50 \%$ [1]. Despite significant efforts to improve pre- and post-operative care, the average survival rates have remained relatively unchanged over the past two decades [1]. New approaches are urgently needed for these patients.

Ex vivo lung tissue engineering has recently emerged as a potential option for increasing the amount of tissue available for transplantation [2]. Lung tissue engineering follows successful clinical approaches using

Provenance: Commissioned article, peer reviewed.

Received: March 062018 | Accepted after revision: May 052018

Copyright CERS 2018. ERR articles are open access and distributed under the terms of the Creative Commons Attribution Non-Commercial Licence 4.0. 
other acellular tissues such as skin [3, 4]. In an ideal scenario, cells would be harvested from the intended transplant recipient, seeded onto a scaffold ex vivo, grown in a bioreactor until the tissue reaches maturity, and finally transplanted (figure 1) [2]. One approach being explored in preclinical models uses a biological scaffold (also called an acellular scaffold) that is derived by decellularising native lung tissue. The goal of any decellularisation protocol is to remove the native cells while preserving both the micro- and macroarchitecture of the organ, as well as the extracellular matrix (ECM) composition and tissue structure [3]. Numerous studies have shown that acellular scaffolds can be derived from a variety of species, including rodents, non-human primates, pigs and humans [5-14].

In parallel, techniques have been developed to generate acellular lung scaffolds originating from animals and also from patients with existing lung diseases [6, 15-21]. These acellular scaffolds retain structural and compositional characteristics of the diseased organs from which they were derived. When cells are reseeded onto these scaffolds, they adopt phenotypes similar to those observed in vivo and thus have emerged as new three-dimensional model systems for studying human disease ex vivo and in particular, cell-matrix interactions.

In this mini-review, we focus on the use of acellular human lung scaffolds as models to study lung repair and regeneration and recent advances in ex vivo lung regeneration for eventual transplantation using acellular human lung scaffolds.

\section{Methods of de- and recellularisation}

The first report of generating and using acellular human lung tissue to study cellular behaviour was reported in 1986 by LweBuga-Mukasa et al. [22]. They decellularised resected segments of human lung tissue from pneumonectomy using a detergent based protocol in order to study the effect of the basement membrane on the morphology and function of type II cells. Following decellularisation, they recellularised the scaffold with freshly isolated rat alveolar type II cells and observed that these cells took on a flattened morphology and lost their lamellar bodies and surface microvilli. They noted that these cells survived up to 8 days ex vivo and underwent morphological flattening and loss of lamellar bodies in this ex vivo system in a similar manner to in vivo alveolar type II to type I differentiation. Interestingly, these morphological changes did not happen as rapidly when cells were cultured on acellular human amniotic basement

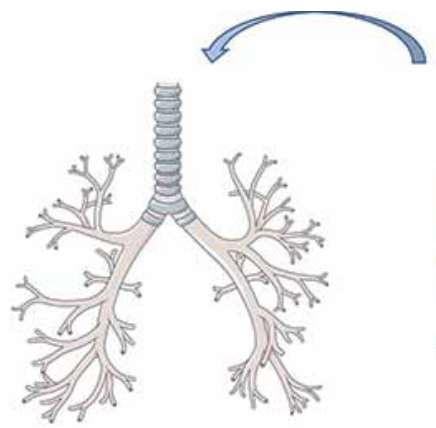

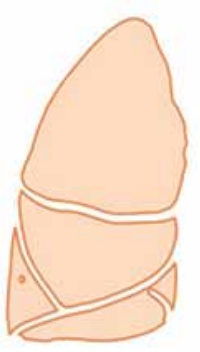

Lung regeneration

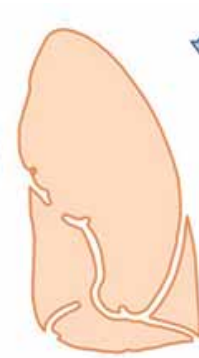

(1)

Tissue assembly

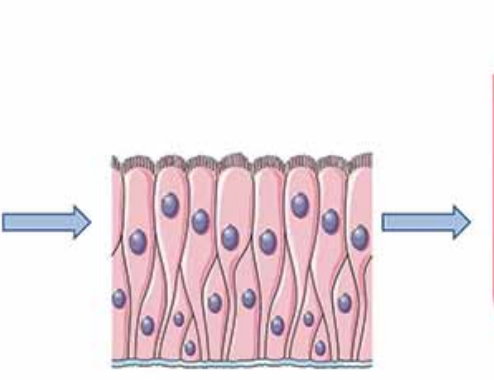

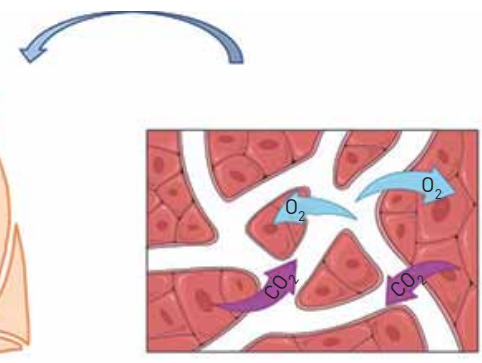

Higher-level tissue function

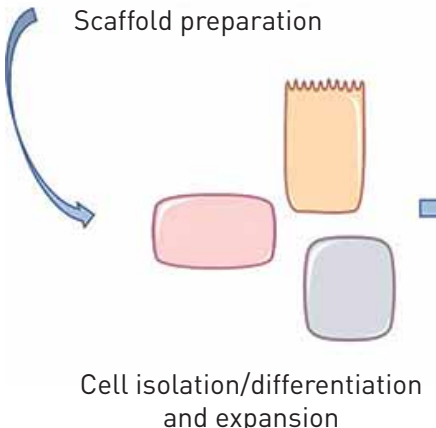
and expansion

FIGURE 1 Schematic outline of the steps in whole lung regeneration. Acellular lung scaffolds are first prepared from donated organs otherwise unsuitable for transplantation. Necessary cell populations must be isolated and/or differentiated and expanded to adequate numbers for scaffold recellularisation. Following delivery of the required cell populations to the correct anatomical positions within the scaffold, the cells must be matured to form organised tissues. This process includes ex vivo culture of the regenerating lung, within specialised bioreactors, which can recapitulate native organ mechanics. Finally, higher-level tissue function, including fluid balance and gas exchange, can be tested both ex vivo and following in vivo implantation in large animal transplantation models. Images reproduced and modified from Servier Medical Art with permission. 
membranes decellularised in a similar manner [23]. Thus, the residual extracellular environment in which these rat alveolar type II cells were cultured had a significant influence on their ex vivo behaviour. It took nearly 25 years before acellular lung scaffolds from human lungs were used as ex vivo models of lung repair and regeneration again.

The techniques used for generating acellular lung scaffolds differ by species and disease status. The majority of protocols rely on a series of detergents used to lyse and remove cells and cell debris, while retaining the ECM composition and structure. There are two main approaches that have been used for decellularisation of lung tissue: 1) perfusion based decellularisation, where decellularisation solutions are perfused through the vasculature and/or airways of the lungs; or 2) immersion based decellularisation, where segments of human lungs are submerged in decellularisation solutions with or without agitation (figure 2). While up-scaling the processes developed in mice to large animal and human lungs has been challenging, several successful protocols now exist for obtaining whole acellular human lung scaffolds [5$11,24]$. Whole acellular lung scaffolds are not needed for all ex vivo models. In fact, for some studies, only certain regions of the lung are of interest. Therefore, techniques have also been developed for decellularisation using immersion based techniques in resected segments of parenchymal lung and bronchi (figure 2) $[15,25,26]$.

There are currently no uniform criteria for what constitutes a decellularised lung. The majority of groups utilise the minimal criteria put forth by CRAPO et al. [3] to define a decellularised tissue: 1) lack of visible nuclei by histological and immunofluorescence staining, 2) $<50 \mathrm{ng}$ of double-stranded DNA per mg tissue dry weight, and 3) DNA fragments $<200 \mathrm{bp}$. However, these criteria do not take into account the mechanical properties of the tissue, sterility of the tissue, the ECM composition or the cytocompatibility of the resulting scaffold. All of these criteria will have an impact on the behaviour of cells re-introduced to the scaffold.

Cytocompatibility is a necessary criterion for any recellularisation study. Owing to difficulties in removing detergents from lungs of larger species, including human lungs, using perfusion based decellularisation approaches, several groups have also evaluated detergent levels during and following decellularisation $[5,24,27]$. This is particularly important to monitor as different cell types have been found to have different thresholds for detergent-induced toxicity [27]. Therefore, differences in residual detergents, which may arise from technical differences (e.g. size of the lung) or differential retention of detergents due to disease state or species, may affect initial cell binding, cell survival and subsequent proliferation. Techniques that do not use detergents may also have residual components that may have an impact on cell survival; thus, each new protocol needs to be thoroughly investigated with regards to cytocompatibility. Achieving both short- and long-term sterility is critical for any tissue engineered scaffold, including the lung. Several studies have evaluated the impact of sterilisation procedures on lung architecture and its impact on recellularisation in animal models and human scaffolds $[5,9,28,29]$. Thus, additional criteria for sterility and cytocompatibility of acellular scaffolds could be as follows: 1) a sterility assurance level of $<10^{-6}$ in the acellular scaffold following any sterilisation procedure [29], and 2) $\geqslant 70 \%$ of cells should survive effluent testing from whole organs or on slices [27]. Fulfilment of both of these criteria is necessary for successful recellularisation.

ECM composition is also an important end-point following decellularisation. Mass spectrometry proteomics has been used to examine differences in the protein composition of acellular human scaffolds using different decellularisation parameters such as choice of detergent or perfusion flow rate [5, 9, 25, 30, 31]. Interestingly, acellular scaffolds derived from normal human lungs retain unique protein compositions [9], independent of the flow rate used for decellularisation. Thus, if a standard decellularisation method is used across a patient cohort, acellular human lung scaffolds can be used to study the heterogeneity that exists between normal patients.

To date, several different methods have been developed for reintroducing cells to acellular human lung scaffolds (termed recellularisation) (figure 2). Following the successful development of techniques to decellularise whole human lungs using perfusion based techniques or segments of human lungs using immersion based techniques, initial studies focused on testing the cytocompatibility of the resulting scaffold. In these studies, cells were either seeded onto thin slices of acellular lung scaffolds or seeded into small segments of acellular lung scaffolds, and initial cell attachment, survival and proliferation were examined over time $[5-7,11,25,32]$. Following the success of these techniques, methods to selectively recellularise through the vasculature or airways of resected segments were then developed [9, 33]. This technique allows the study of more specific cell-ECM interactions (e.g. endothelial basement membrane versus epithelial basement membrane), rather than relying on stochastic cell adhesion when cells are exposed to the whole scaffold. Regardless of the technique used for recellularisation, these early studies importantly confirmed that the techniques used for decellularisation yielded cytocompatible scaffolds. Acellular human lung parenchyma or bronchial scaffolds [26] have been shown to provide support for cell 
survival and proliferation of several different types of primary and immortalised cell lines for up to 35 days ex vivo (table 1), making them suitable models for studying both short- and longer-term processes.

In addition to recellularisation into segments or onto thin slices, techniques to homogenise and solubilise the ECM derived following decellularisation have been developed and allow the study of cell-ECM

Native lung (normal or diseased)

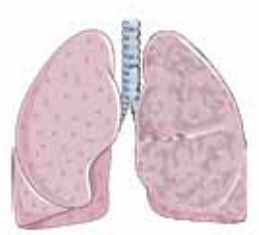

Perfusion decellularisation
Resections
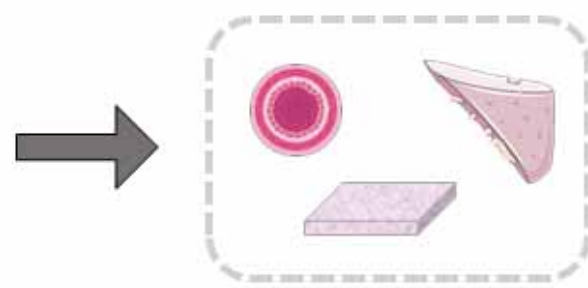

- Bronchus

- Parenchyma

Acellular scaffold

Immersion

decellularisation
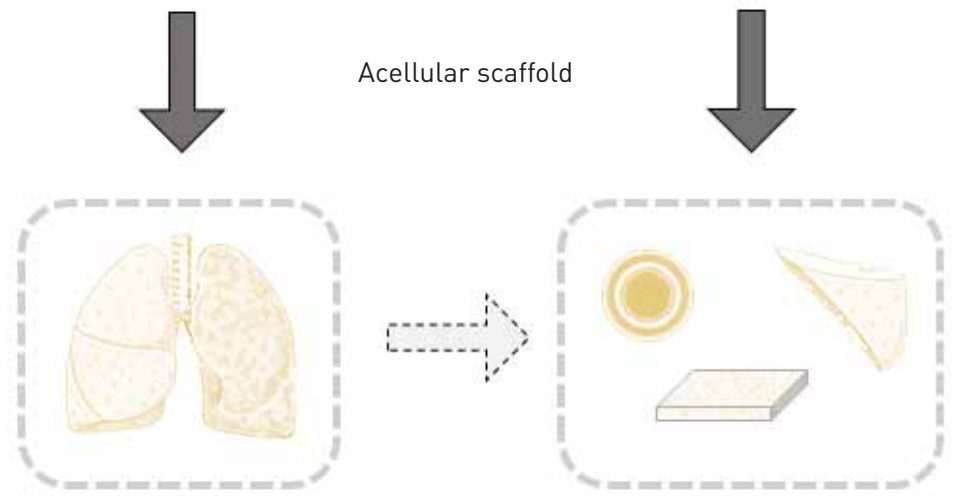

ECM extraction
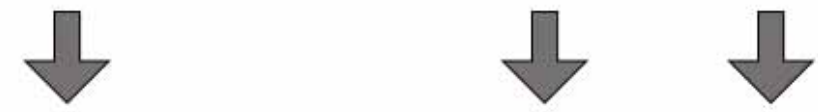

Whole lobe/lung

$3 \mathrm{D}$ segment

Thin slice
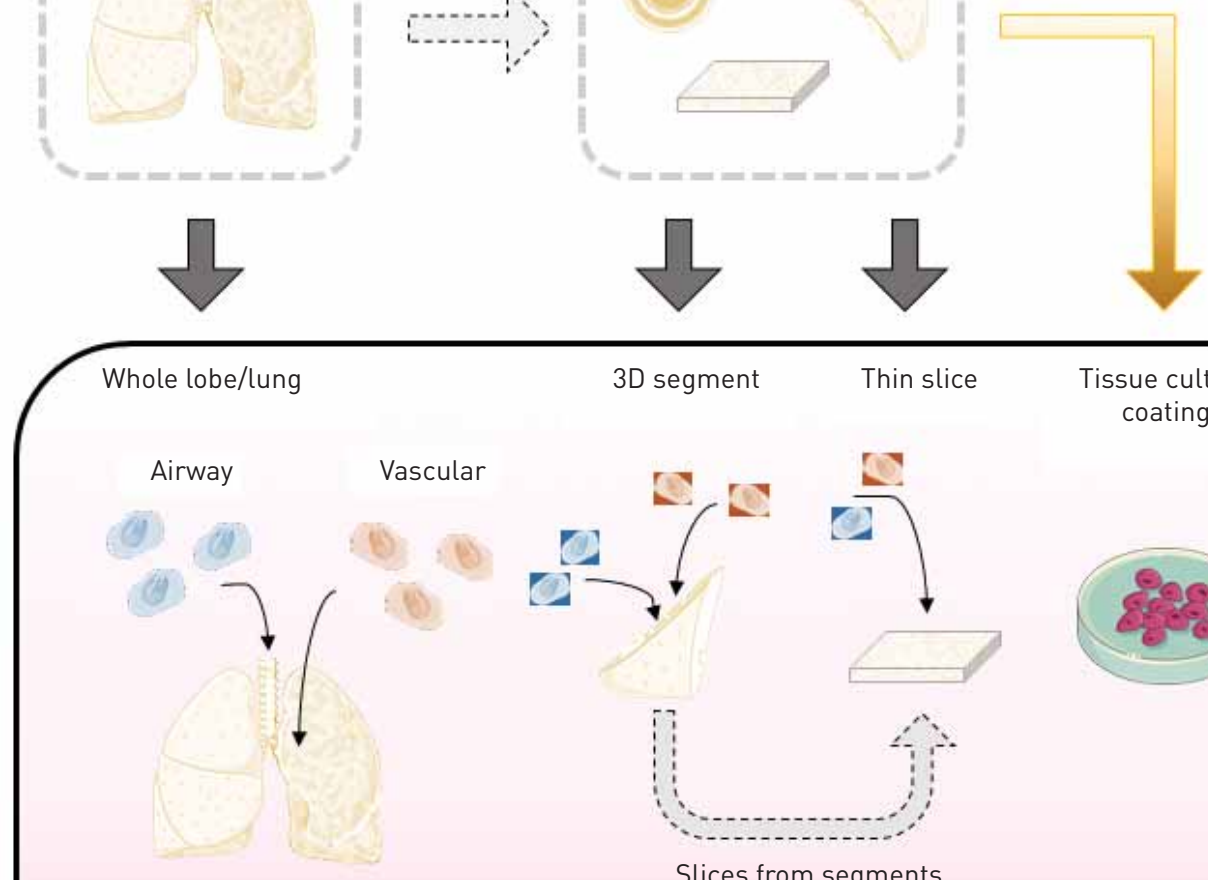

Recellularisation

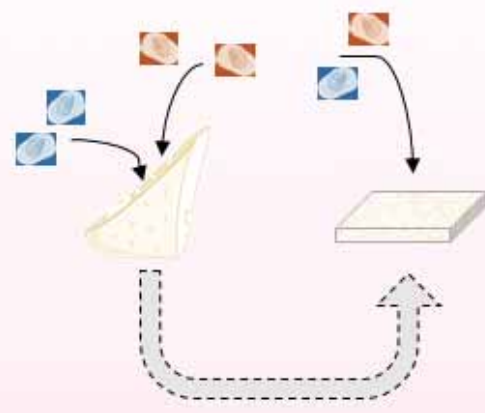

Slices from segments

FIGURE 2 Overview of techniques to generate acellular human lung scaffolds for modelling lung disease repair and regeneration. Acellular scaffolds may be obtained from normal or diseased human lungs using either perfusion or immersion based decellularisation. Following successful decellularisation, cells may be reseeded into whole lungs or lobes, seeded into small three-dimensional (3D) segments, or seeded onto thin slices. Recellularised small 3D segments may be left intact or further processed into thin slices. Alternatively, extracellular matrix (ECM) may be extracted from acellular human lung tissue and used to coat cell culture dishes. Images reproduced and modified from Servier Medical Art with permission. 
TABLE 1 Comprehensive list of studies using acellular normal or diseased human lung tissue

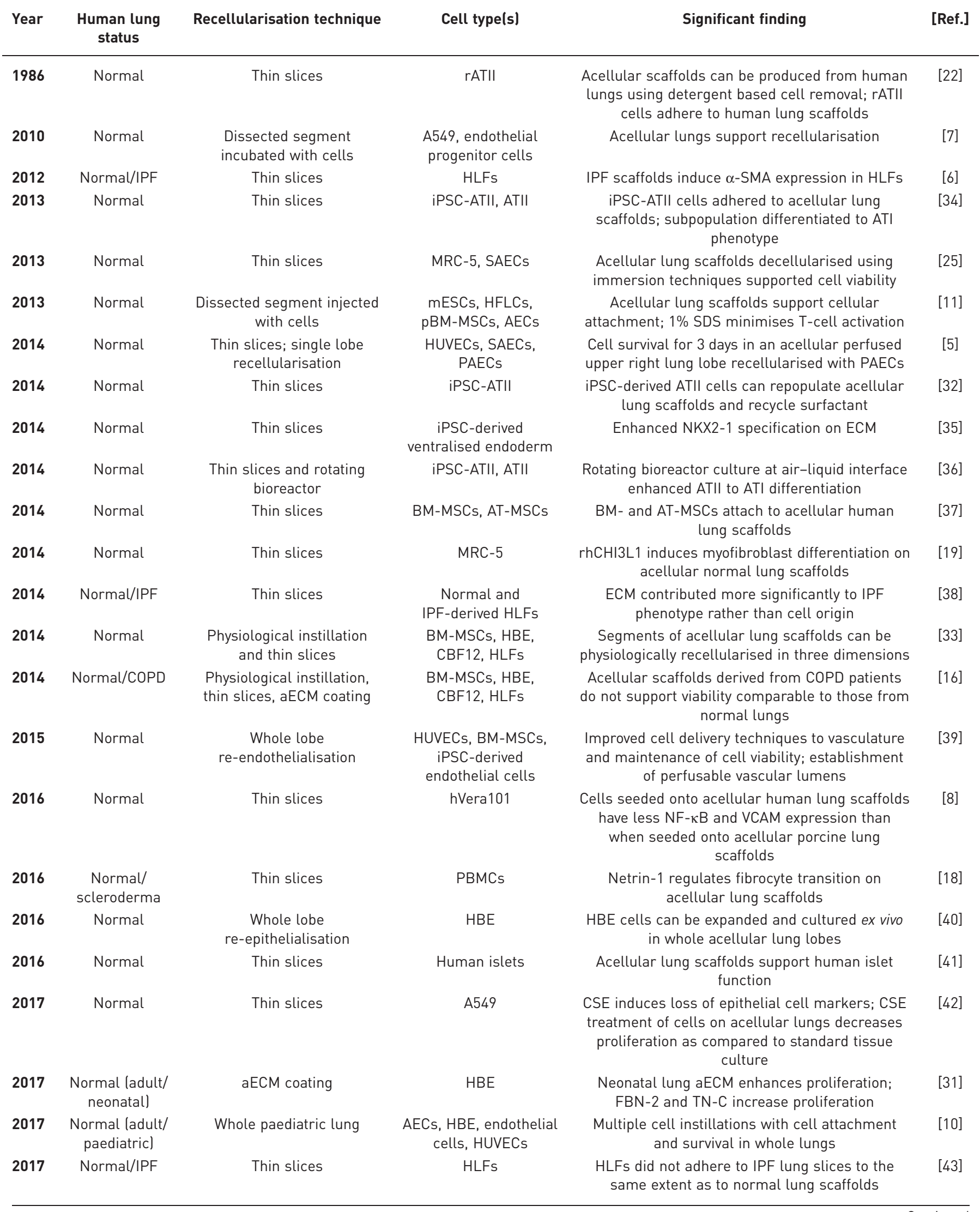




\begin{tabular}{|c|c|c|c|c|c|}
\hline Year & $\begin{array}{l}\text { Human lung } \\
\text { status }\end{array}$ & Recellularisation technique & Cell type(s) & Significant finding & [Ref.] \\
\hline 2018 & Normal/COPD & Floating bronchial rings & HBE & $\begin{array}{l}\text { Bronchial ECM from COPD patients induces } \\
\text { differential gene expression in primary HBE cells }\end{array}$ & {$[26]$} \\
\hline 2018 & Normal/IPF & Thin slices & SAECs & $\begin{array}{c}\text { Acellular scaffolds derived from IPF lungs induce } \\
\text { loss of the epithelial phenotype }\end{array}$ & [15] \\
\hline \multicolumn{6}{|c|}{$\begin{array}{l}\text { rATII: rat alveolar type II cells; IPF: idiopathic pulmonary fibrosis; HLF: human lung fibroblast; } \alpha \text {-SMA: } \alpha \text {-smooth muscle actin; iPSC: human } \\
\text { induced pluripotent stem cell; ATII: alveolar type II; ATI: alveolar type I; MRC-5: Medical Research Council cell strain 5, human fibroblast cell } \\
\text { line; SAEC: human small airway epithelial cell; mESC: murine embryonic stem cell; HFLC: human fetal lung cell; pBM-MSC: porcine bone } \\
\text { marrow-derived mesenchymal stem cell; AEC: human alveolar epithelial cell; HUVEC: human umbilical vein endothelial cell; PAEC: pulmonary } \\
\text { alveolar epithelial cell; NKX2-1: NK2 homeobox 1; ECM: extracellular matrix; BM-MSC: human bone marrow-derived mesenchymal stem cell; } \\
\text { AT-MSC: human adipose tissue-derived mesenchymal stem cell; rhCHI3L1: recombinant human chitinase 3-like 1; HBE: human bronchial } \\
\text { epithelial; CBF12: human endothelial progenitor cells; COPD: chronic obstructive pulmonary disease; aECM: ECM coatings for tissue culture } \\
\text { plastic derived from acellular lungs; hVera101: human VeraVec cells, a modified umbilical cord endothelial cell line; VCAM: vascular cell } \\
\text { adhesion molecule; PBMC: peripheral blood mononuclear cell; CSE: cigarette smoke extract; FBN-2: fibrillin-2; TN-C: tenascin-C; PC: human } \\
\text { microvascular pericyte. }\end{array}$} \\
\hline
\end{tabular}

interactions in a high-throughput manner. To date, pepsin digestion and acid extraction have been used to obtain ECM solutions that can be used to coat tissue culture wells or polyacrylamide hydrogels of different stiffness $[16,17,31]$. These techniques have already been used to study a variety of cellular behaviours, such as cell adhesion to ECM components, cell viability, proliferation and cell migration, and to decouple mechanical changes in the ECM from its composition.

\section{Use of acellular human scaffolds to study repair and disease}

In parallel to the advances made for regenerating lung tissue ex vivo using acellular scaffolds, methods have been developed to generate acellular scaffolds derived from patients with chronic lung disease (table 1) $[6,16-18]$. These techniques have opened new doors for studying cell-ECM interactions and have provided new insights into potential disease pathomechanisms, especially for chronic diseases where in vivo animal models do not adequately recapitulate human disease. Both disease and the age of the patient from which the acellular lung scaffold originates have been shown to affect initial cell adhesion, proliferation and survival of seeded cells $[16,31,43]$.

In addition to histological characterisation using standard techniques, several groups have used techniques such as second harmonic generation and mass spectrometry based proteomics to characterise acellular scaffolds derived from chronic lung disease patients $[6,16,18,43]$. In general, ECM components are difficult to detect using mass spectrometry based proteomics, owing to the dramatic differences in protein solubility between cell-associated proteins and ECM-associated proteins. Decellularisation [44] and other detergent solubilisation techniques that mimic decellularisation [45] have opened up new possibilities for examining differences in human lung ECM using mass spectrometry. Several studies have examined differences in the ECM derived from patients with chronic lung diseases, such as idiopathic pulmonary fibrosis [6] and emphysema [16], as well as from patients of different ages (neonatal lungs versus adult lungs) [31]. These studies have yielded important insights into differences in individual components within the ECM, such as major collagens, laminins and glycoproteins, which may be driving the phenotypic differences observed when cells are cultured on these scaffolds.

Protocols for decellularising whole human lungs or individual lobes may need to be adapted depending on the disease status of the patient. While protocols to decellularise individual lobes from normal patients could be used for patients with emphysema [16], these same protocols were found to be inadequate for patients with ILD $[9,15]$. ILD causes altered tissue-level mechanics and thus the flow of decellularisation reagents throughout the tissue is altered when perfusion based decellularisation is used. Furthermore, even if segments are resected from these lungs, the parenchyma is extensively remodelled in ILD, resulting in heterogeneous regions of dense ECM. Thus, detergents may need to be used at higher concentrations or for longer periods of time to ensure adequate decellularisation compared to tissue derived from normal lungs [15].

Differences observed using the same decellularisation protocol in normal and diseased human lungs may be a result of tissue-level changes in disease but may also reflect differences in extractability of different 
ECM components in disease, which could be due to changes in crosslinking frequency or disease-related degradation or secretion [46]. Due to the inherent heterogeneity of human samples, it is important that enough patients are included to overcome this variability and yield meaningful results. Furthermore, as multiple investigators, both in the lung field and in other tissues, have identified a large variety of non-ECM proteins in acellular human and animal scaffolds [9, 44], it is important for investigators using these ex vivo models to understand the limitations of using acellular scaffolds to study cell-ECM interactions. The effect of residual proteins, such as growth factors and other cell-associated proteins such as histones and cytoskeletal proteins, may not be negligible in these settings and may induce responses that do not correspond to in vivo environments [47]. Thus, while acellular human lung scaffolds are a powerful ex vivo system for studying cell behaviour in a three-dimensional environment and in many ways better reflect clinical heterogeneity and human disease compared to using animals that are genetically similar, it is important that the aforementioned limitations are taken into account when designing experiments and interpreting results.

\section{Bioengineering: recellularisation of whole lung scaffolds}

The bigger goal of utilising native ECM scaffolds to regenerate functional lung tissue still faces many questions and challenges. Successful recellularisation of these scaffolds will require the isolation and expansion of the necessary cell populations for tissue regeneration. Two main cell sources currently being investigated are 1) primary tissue-isolated progenitor cells [40] and 2) differentiated pluripotent stem cells [39, 48-50].

Primary cells provide a useful tool to study and develop effective lung recellularisation strategies. Cell lines including human umbilical vein endothelial cells and A549 alveolar epithelial cells have been used in numerous experiments to demonstrate biocompatibility to the decellularised scaffold. While useful, these cells possess less translational potential as they are not patient specific and may not be derived from normal tissues. Lung tissue-isolated cell populations have also been used in recellularisation studies. As a tissue that is under constant assault from the external environment, the lung possesses several endogenous progenitor cell populations that can be activated to regenerate tissue following injury or damage [51]. Emerging research has demonstrated that some of these progenitor cells may be capable of multi-lineage differentiation, contributing to both proximal and distal lung regeneration [52, 53], which could be exploited in complex lung tissue recellularisation. However, isolation and expansion of these rare cell types in sufficient numbers remains a barrier to their utility in whole organ recellularisation. Also, lung diseases underlying the need for transplantation will probably limit the function and expansion capacity of these primary cells. The use of induced pluripotent stem cell (iPSC)-derived cell populations has the potential to overcome the need for primary lung tissue. A terminally differentiated cell of any origin could, in theory, be reprogrammed to the pluripotent state and subsequently differentiated to numerous lung-specific cell types. Challenges in the efficiency, specificity and scalability of iPSC-differentiation protocols remain, and the issue of inherent diseases may still need to be solved. Donor-specific genetic diseases, such as cystic fibrosis, may have the possibility to be corrected in the iPSC state, prior to differentiation, although maintaining the genome integrity of the iPSCs and the differentiated cells is still an uncertainty [54]. The idea of a universal donor tissue or bank of tissues, from which iPSCs can be generated and differentiated to match donors regardless of tissue type, is an exciting potential solution to this problem [55], but remains under development.

Even with adequate numbers of the correct cell populations, efficient repopulation of the scaffolds in a site-specific manner poses another challenge. This will require methods to organise and pattern the redelivered cells into the three-dimensional structure of the acellular scaffold. Building upon the native structure, mature cells will either need to be directed to the correct anatomical space (e.g. proximal versus distal lung epithelium) or be delivered in a progenitor state and targeted to specifically differentiate once in the lung scaffold. The microenvironment, including growth factors, local oxygen tension, cell-cell interactions and dynamic tissue mechanics, can be manipulated to direct higher-level tissue organisation $[56,57]$. This process will require the design of specific bioreactors to provide the optimal regenerative environment for ex vivo tissue maturation. For lung bioengineering, this includes vascular perfusion, the capacity for fluid or air ventilation, and the ability to test functional end-points of tissue regeneration, including gas exchange, cellular metabolism and barrier function [58]. The ability to recapitulate key developmental cues to the regenerating organ is a main advantage of custom-designed bioreactors, which will aid in the creation of optimised and targeted organ culture protocols that drive innate repair mechanisms.

\section{Translation to clinically relevant, transplantable grafts}

A major aim of the field is to create therapeutic solutions for end-organ failure and alleviate the current donor shortage, making lung transplantation a patient-specific, on-demand process. Progress toward this 
goal has utilised porcine scaffolds, recellularised with human cell populations, followed by ex vivo culture and re-implantation to porcine recipients $[2,59]$. Decellularised porcine scaffolds were repopulated with human umbilical vein endothelial cells in the vascular network and basal epithelial stem cells in the airways. Following 6 days of ex vivo culture, the constructs were transplanted, and vascular reperfusion and ventilation re-established, for $1 \mathrm{~h}$ of functional assessment. Basal gas exchange was achieved, although an immature barrier function and lack of pulmonary surfactant limited overall function of the graft. Challenges in vascular perfusability and coagulation also remain in the path towards the goal of successfully engineering a graft with long-term survival. Important questions regarding the necessity of other cell types, as well as the innate and acquired immune system response to engineered tissues based on either human or xenogeneic scaffolds, must be addressed once extended graft survival is achieved [60]. As advances in bioengineering methods are achieved and graft function improves, extensive preclinical testing in large animal models will be an important requirement towards human transplantation. Due to the potential for large patient-to-patient heterogeneity and complexity in translational parameters (e.g. scaffold source, cell source and ex vivo culturing parameters), sufficient numbers of studies will be required to thoroughly validate any clinical-grade procedure and effectively translate this technology to a therapy.

\section{Conclusion}

The creation and use of acellular lung scaffolds has provided important new tools and opportunities for studying lung repair and regeneration, and furthermore provides a more physiological setting for studying human lung diseases. If the goal of utilising ex vivo lung bioengineering approaches to create functional, transplantable grafts can be realised, this approach has the potential to overcome the challenges of organ donor shortages and prevent allograft immune rejection. Ultimately, progress in the fields of developmental biology, cell biology, physiology and transplantation can be combined to create therapeutic solutions for lung disease and end-organ failure.

Acknowledgements: The authors wish to thank Deniz Bölükbas and Martina de Santis (both Lund University, Lund, Sweden) for helpful feedback on the figures.

Conflict of interest: S.E. Gilpin reports a grant from the Francis Family Foundation (a Parker B. Francis Fellowship), during the conduct of the study; in addition, S.E. Gilpin has a patent pending on Human Airway Stem Cells in Lung Epithelial Engineering (number 20170326273). D.E. Wagner reports a grant from the Knut and Alice Wallenberg Foundation, during the conduct of the study; in addition, D.E. Wagner has a patent pending on System and Method for Decellularization and Recellularization of Whole Organs (US Provisional Patent Application No. 61/810,966).

Support statement: This work was funded by a Wallenberg Molecular Medicine Fellowship (to D.E. Wagner) from the Knut and Alice Wallenberg Foundation, and a Parker B. Francis Fellowship (to S.E. Gilpin) from the Francis Family Foundation. Funding information for this article has been deposited with the Crossref Funder Registry.

\section{References}

1 Yusen RD, Edwards LB, Kucheryavaya AY, et al. The Registry of the International Society for Heart and Lung Transplantation: Thirty-second Official Adult Lung and Heart-Lung Transplantation Report - 2015. J Heart Lung Transplant 2015; 34: 1264-1277.

2 Gilpin SE, Charest JM, Ren X, et al. Bioengineering lungs for transplantation. Thorac Surg Clin 2016; 26: 163-171.

3 Crapo PM, Gilbert TW, Badylak SF. An overview of tissue and whole organ decellularization processes. Biomaterials 2011; 32: 3233-3243.

4 Wainwright DJ. Use of an acellular allograft dermal matrix (AlloDerm) in the management of full-thickness burns. Burns 1995; 21: 243-248.

5 Gilpin SE, Guyette JP, Gonzalez G, et al. Perfusion decellularization of human and porcine lungs: bringing the matrix to clinical scale. J Heart Lung Transplant 2014; 33: 298-308.

6 Booth AJ, Hadley R, Cornett AM, et al. Acellular normal and fibrotic human lung matrices as a culture system for in vitro investigation. Am J Respir Crit Care Med 2012; 186: 866-876.

7 Petersen TH, Calle EA, Zhao L, et al. Tissue-engineered lungs for in vivo implantation. Science 2010; 329: 538-541.

8 Balestrini JL, Gard AL, Gerhold KA, et al. Comparative biology of decellularized lung matrix: implications of species mismatch in regenerative medicine. Biomaterials 2016; 102: 220-230.

9 Wagner DE, Bonenfant NR, Sokocevic D, et al. Three-dimensional scaffolds of acellular human and porcine lungs for high throughput studies of lung disease and regeneration. Biomaterials 2014; 35: 2664-2679.

10 Nichols JE, La Francesca S, Vega SP, et al. Giving new life to old lungs: methods to produce and assess whole human paediatric bioengineered lungs. J Tissue Eng Regen Med 2017; 11: 2136-2152.

11 Nichols JE, Niles J, Riddle M, et al. Production and assessment of decellularized pig and human lung scaffolds. Tissue Eng Part A 2013; 19: 2045-2062.

12 Bonvillain RW, Danchuk S, Sullivan DE, et al. A nonhuman primate model of lung regeneration: detergent-mediated decellularization and initial in vitro recellularization with mesenchymal stem cells. Tissue Eng Part A 2012; 18: 2437-2452.

13 Price AP, England KA, Matson AM, et al. Development of a decellularized lung bioreactor system for bioengineering the lung: the matrix reloaded. Tissue Eng Part A 2010; 16: 2581-2591.

14 Ott HC, Clippinger B, Conrad C, et al. Regeneration and orthotopic transplantation of a bioartificial lung. Nat Med 2010; 16: 927-933. 
15 van der Velden JL, Wagner DE, Lahue KG, et al. TGF- $\beta 1$-induced deposition of provisional extracellular matrix by tracheal basal cells promotes epithelial-to-mesenchymal transition in a JNK1-dependent manner. Am J Physiol Lung Cell Mol Physiol 2018; in press [https://doi.org/10.1152/ajplung.00053.2017].

16 Wagner DE, Bonenfant NR, Parsons CS, et al. Comparative decellularization and recellularization of normal versus emphysematous human lungs. Biomaterials 2014; 35: 3281-3297.

17 Sava P, Ramanathan A, Dobronyi A, et al. Human pericytes adopt myofibroblast properties in the microenvironment of the IPF lung. JCI Insight 2017; 2: e96352.

18 Sun $\mathrm{H}$, Zhu Y, Pan H, et al. Netrin-1 regulates fibrocyte accumulation in the decellularized fibrotic sclerodermatous lung microenvironment and in bleomycin-induced pulmonary fibrosis. Arthritis Rheumatol 2016; 68: 1251-1261.

19 Zhou Y, Peng H, Sun H, et al. Chitinase 3-like 1 suppresses injury and promotes fibroproliferative responses in mammalian lung fibrosis. Sci Transl Med 2014; 6: 240 ra76.

20 Sokocevic D, Bonenfant NR, Wagner DE, et al. The effect of age and emphysematous and fibrotic injury on the re-cellularization of de-cellularized lungs. Biomaterials 2013; 34: 3256-3269.

21 Scarritt ME, Bonvillain RW, Burkett BJ, et al. Hypertensive rat lungs retain hallmarks of vascular disease upon decellularization but support the growth of mesenchymal stem cells. Tissue Eng Part A 2014; 20 : 1426-1443.

22 Lwebuga-Mukasa JS, Ingbar DH, Madri JA. Repopulation of a human alveolar matrix by adult rat type II pneumocytes in vitro. A novel system for type II pneumocyte culture. Exp Cell Res 1986; 162: 423-435.

23 Lwebuga-Mukasa JS, Thulin G, Madri JA, et al. An acellular human amnionic membrane model for in vitro culture of type II pneumocytes: the role of the basement membrane in cell morphology and function. $J$ Cell Physiol 1984; 121: 215-225.

24 Price AP, Godin LM, Domek A, et al. Automated decellularization of intact, human-sized lungs for tissue engineering. Tissue Eng Part C Methods 2015; 21: 94-103.

25 O’Neill JD, Anfang R, Anandappa A, et al. Decellularization of human and porcine lung tissues for pulmonary tissue engineering. Ann Thorac Surg 2013; 96: 1046-1055.

26 Hedström U, Hallgren O, Öberg L, et al. Bronchial extracellular matrix from COPD patients induces altered gene expression in repopulated primary human bronchial epithelial cells. Sci Rep 2018; 8: 3502.

27 Zvarova B, Uhl FE, Uriarte JJ, et al. Residual detergent detection method for nondestructive cytocompatibility evaluation of decellularized whole lung scaffolds. Tissue Eng Part C Methods 2016; 22: 418-428.

28 Bonenfant NR, Sokocevic D, Wagner DE, et al. The effects of storage and sterilization on de-cellularized and re-cellularized whole lung. Biomaterials 2013; 34: 3231-3245.

29 Balestrini JL, Liu A, Gard AL, et al. Sterilization of lung matrices by supercritical carbon dioxide. Tissue Eng Part C Methods 2016; 22: 260-269.

30 Wallis JM, Borg ZD, Daly AB, et al. Comparative assessment of detergent-based protocols for mouse lung de-cellularization and re-cellularization. Tissue Eng Part C Methods 2012; 18: 420-432.

31 Gilpin SE, Li Q, Evangelista-Leite D, et al. Fibrillin-2 and Tenascin-C bridge the age gap in lung epithelial regeneration. Biomaterials 2017; 140: 212-219.

32 Huang SX, Islam MN, O'Neill J, et al. Efficient generation of lung and airway epithelial cells from human pluripotent stem cells. Nat Biotech 2014; 32: 84-91.

33 Wagner DE, Fenn SL, Bonenfant NR, et al. Design and synthesis of an artificial pulmonary pleura for high throughput studies in acellular human lungs. Cell Mol Bioeng 2014; 7: 184-195.

34 Ghaedi M, Calle EA, Mendez JJ, et al. Human iPS cell-derived alveolar epithelium repopulates lung extracellular matrix. J Clin Invest 2013; 123: 4950-4962.

35 Gilpin SE, Ren X, Okamoto T, et al. Enhanced lung epithelial specification of human induced pluripotent stem cells on decellularized lung matrix. Ann Thorac Surg 2014; 98: 1721-1729.

36 Ghaedi M, Mendez JJ, Bove PF, et al. Alveolar epithelial differentiation of human induced pluripotent stem cells in a rotating bioreactor. Biomaterials 2014; 35: 699-710.

37 Mendez JJ, Ghaedi M, Steinbacher D, et al. Epithelial cell differentiation of human mesenchymal stromal cells in decellularized lung scaffolds. Tissue Eng Part A 2014; 20: 1735-1746.

38 Parker MW, Rossi D, Peterson M, et al. Fibrotic extracellular matrix activates a profibrotic positive feedback loop. J Clin Invest 2014; 124: 1622-1635.

39 Ren X, Moser PT, Gilpin SE, et al. Engineering pulmonary vasculature in decellularized rat and human lungs. Nat Biotechnol 2015; 33: 1097-1102.

40 Gilpin SE, Charest JM, Ren X, et al. Regenerative potential of human airway stem cells in lung epithelial engineering. Biomaterials 2016; 108: 111-119.

41 Abualhassan N, Sapozhnikov L, Pawlick RL, et al. Lung-derived microscaffolds facilitate diabetes reversal after mouse and human intraperitoneal islet transplantation. PLoS One 2016; 11: e0156053.

42 Bruzauskaite I, Raudoniute J, Denkovskij J, et al. Native matrix-based human lung alveolar tissue model in vitro: studies of the reparatory actions of mesenchymal stem cells. Cytotechnology 2017; 69: 1-17.

43 Tjin G, White ES, Faiz A, et al. Lysyl oxidases regulate fibrillar collagen remodelling in idiopathic pulmonary fibrosis. Dis Model Mech 2017; 10: 1301-1312.

44 Li Q, Uygun BE, Geerts S, et al. Proteomic analysis of naturally-sourced biological scaffolds. Biomaterials 2016; 75: 37-46.

45 Schiller HB, Mayr CH, Leuschner G, et al. Deep proteome profiling reveals common prevalence of MZB1-positive plasma B cells in human lung and skin fibrosis. Am J Respir Crit Care Med 2017; 196: 1298-1310.

46 Åhrman E, Hallgren O, Malmström L, et al. Quantitative proteomic characterization of the lung extracellular matrix in chronic obstructive pulmonary disease and idiopathic pulmonary fibrosis. J Proteomics 2018; in press [https://doi.org/10.1016/j.jprot.2018.02.027].

47 Dziki JL, Huleihel L, Scarritt ME, et al. Extracellular matrix bioscaffolds as immunomodulatory biomaterials Tissue Eng Part A 2017; 23: 1152-1159.

48 Huang SX, Green MD, de Carvalho AT, et al. The in vitro generation of lung and airway progenitor cells from human pluripotent stem cells. Nat Protoc 2015; 10: 413-425.

49 Jacob A, Morley M, Hawkins F, et al. Differentiation of human pluripotent stem cells into functional lung alveolar epithelial cells. Cell Stem Cell 2017; 21: 472-488.e10. 
50 McCauley KB, Hawkins F, Serra M, et al. Efficient derivation of functional human airway epithelium from pluripotent stem cells via temporal regulation of Wnt signaling. Cell Stem Cell 2017; 20: 844-857.e6.

51 Kotton DN, Morrisey EE. Lung regeneration: mechanisms, applications and emerging stem cell populations. Nat Med 2014; 20: 822-832.

52 Zuo W, Zhang T, Wu DZ, et al. $\mathrm{p} 63^{+} \mathrm{Krt}^{+}$distal airway stem cells are essential for lung regeneration. Nature 2015; 517: 616-620.

53 Vaughan AE, Brumwell AN, Xi Y, et al. Lineage-negative progenitors mobilize to regenerate lung epithelium after major injury. Nature 2015; 517: 621-625.

54 Jang YY, Ye Z. Gene correction in patient-specific iPSCs for therapy development and disease modeling. Hum Genet 2016; 135: 1041-1058.

55 Tsukamoto A, Abbot SE, Kadyk LC, et al. Challenging regeneration to transform medicine. Stem Cells Transl Med 2016; 5: 1-7.

56 Nonaka PN, Uriarte JJ, Campillo N, et al. Lung bioengineering: physical stimuli and stem/progenitor cell biology interplay towards biofabricating a functional organ. Respir Res 2016; 17: 161.

57 Garreta E, Melo E, Navajas D, et al. Low oxygen tension enhances the generation of lung progenitor cells from mouse embryonic and induced pluripotent stem cells. Physiol Rep 2014; 2: e12075.

58 Charest JM, Okamoto T, Kitano K, et al. Design and validation of a clinical-scale bioreactor for long-term isolated lung culture. Biomaterials 2015; 52: 79-87.

59 Zhou H, Kitano K, Ren X, et al. Bioengineering human lung grafts on porcine matrix. Ann Surg 2018; 267: 590-598.

60 Wiles K, Fishman JM, De Coppi P, et al. The host immune response to tissue-engineered organs: current problems and future directions. Tissue Eng Part B Rev 2016; 22: 208-219. 